\title{
EIC Message
}

As I start my second three-year term as Editor-in-Chief (EiC) of ACM TOS, I would like to take this opportunity to announce some shuffling of Associate Editors. Those leaving are (in alphabetical order) Nitin Agrawal, Sangyeon Cho, Cheng Huang, Onur Mutlu, Michael Swift, Nisha Talagala, Andy Wang, and Tong Zhang. I thank them for their devoted services the last three years. Without their sacrifice, it would have been impossible to run this journal. I am also appointing a new batch of Associate Editors. Namely (again, alphabetically), Yuan Hao Chang, Jooyoung Hwang, Geoff Kuenning, Philip Shilane, Devesh Tiwali, Swami Sundararaman, and Ming Zhao. As their short bios that follow shows, they are all respected experts in the field of storage. I am sure they will contribute immensely to the continued success of our journal.

Yuan-Hao Chang is currently a Research Fellow (Professor) and Deputy Director of Institute of Information Science (IIS), Academia Sinica, Taipei, Taiwan. He has been an associate editor of IEEE Transactions on Emerging Topics in Computing (TETC) and ACM Transactions on CyberPhysical Systems (TCPS) and has also served as a program committee member for many top international conferences (e.g., ACM/IEEE DAC, ACM/IEEE ISLPED, IEEE RTSS, IEEE RTAS, ACM/IEEE CODES+ISSS, and IEEE ICDCS). His research interests include memory/storage systems, operating systems, embedded systems, and real-time systems.

Jooyoung Hwang is a VP for research in Samsung Electronics memory business unit. He is responsible for research and development of embedded software of solid state drives and storage system software. His research interests include file systems, solid state drive technologies, and high-performance storage. He holds a $\mathrm{PhD}$ in electrical engineering from Korea Advanced Institute of Science and Technology (KAIST).

Geoff Kuenning is a Professor of Computer Science at Harvey Mudd College in Claremont, California. After receiving a B.S. and M.S. in Computer Science from Michigan State University in the early 1970s, he worked in the industry for 15 years before beginning PhD studies at UCLA in 1989. Since joining Harvey Mudd in 1998, he has participated in the rapid growth of the Computer Science Department, including helping to increase female participation in the major from $10 \%$ to $50 \%$. He also served as Computer Science Clinic Director for six years. His research interests include operating systems and file systems, and he is the primary force behind the SNIA IOTTA Trace Repository, which collects I/O trace datasets and makes them available to researchers around the world.

Devesh Tiwali leads the Goodwill Computing Lab (https://web.northeastern.edu/tiwari/) at Northeastern University, where his group focuses on innovating new methods to improve efficiency, scalability, and cost-effectiveness of large-scale parallel computing \& storage systems. Before joining Northeastern, Devesh was a staff scientist at the Oak Ridge National Laboratory-the United States Department of Energy (DOE) Laboratory. Devesh earned his PhD in Electrical and Computer Engineering from North Carolina State University and B.S. degree in Computer Science and Engineering from the Indian Institute of Technology (IIT) Kanpur in India.

Philip Shilane received B.S. and M.S. degrees in computer science from Stanford University in Stanford, California, in 2000 and 2001, respectively; and M.A. and PhD degrees in computer

(C) 2020 Copyright held by the owner/author(s).

1553-3077/2020/01-ART22e

https://doi.org/10.1145/3372345

ACM Transactions on Storage, Vol. 15, No. 4, Article 22e. Publication date: January 2020. 
science from Princeton University in Princeton, New Jersey, in 2004 and 2008, respectively. Since 2007, he has worked for Data Domain, EMC, and Dell Technologies in research and advanced development within a CTO organization in the areas of computer storage systems, deduplication, compression, data characterization, flash caching, and cloud microservices. Philip was recognized in 2019 as a Distinguished Member of Technical Staff within Dell Technologies. He has more than 30 publications in journals and conferences, including ACM Transactions on Storage, Proceedings of the IEEE, USENIX ATC, USENIX FAST, Middleware, Massive Storage Systems and Technology, USENIX HotStorage, ACM Transactions on Graphics, SIGGRAPH, Shape Modeling International, and so on, and he regularly serves on conference program committees and reviews journal articles. He is an inventor of over 80 patents.

Swaminathan (Swami) Sundararaman is the CTO at Pyxeda.ai and was previously the Lead Architect of ParallelM, an early-stage startup focused on Production Machine Learning and Deep Learning. He also worked at Fusion-io Inc. and Sandisk Corp., where he focused on innovations in nonvolatile memory technologies and applications. He holds a $\mathrm{PhD}$ from the University of Wisconsin-Madison and holds 15 patents in Distributed Systems, Operating Systems, Non-Volatile Memory, and Storage Systems. Swami co-founded the OpML and HotEdge conferences that foster upcoming innovations in operationalizing ML/DL models in production and edge computing, respectively. He serves on the steering committee and program committees of multiple technical conferences. His research interests include distributed systems, machine learning, edge computing, operating systems, and file storage systems.

Ming Zhao is an associate professor of the Arizona State University (ASU) School of Computing, Informatics, and Decision Systems Engineering (CIDSE), where he directs the research laboratory for Virtualized Infrastructures, Systems, and Applications (VISA, http://visa.lab.asu.edu). His research is in the areas of experimental computer systems, including cloud/edge, big-data, and high-performance systems as well as operating systems and storage in general. He is also interested in the interdisciplinary studies that bridge computer systems research with other domains. His research outcomes have been adopted by several production systems. Dr. Zhao received his bachelor's and master's degrees from Tsinghua University and his $\mathrm{PhD}$ from University of Florida. 